\title{
Importance of retinoic acid-inducible gene $I$ and of receptor for type $I$ interferon for cellular resistance to infection by Newcastle disease virus
}

\author{
PHILIPPE FOURNIER $^{1}$, HOLGER WILDEN ${ }^{1}$ and VOLKER SCHIRRMACHER ${ }^{1,2}$ \\ ${ }^{1}$ German Cancer Research Center, Im Neuenheimer Feld 280, D-69120 Heidelberg; ${ }^{2}$ Center of Immunology \\ and Oncology (IOZK), Hohenstaufenring 30-32, D-50674 Cologne, Germany
}

Received August 6, 2011; Accepted September 1, 2011

DOI: $10.3892 /$ ijo.2011.1222

\begin{abstract}
Newcastle disease virus (NDV) is an avian paramyxovirus with oncolytic properties which shows promising effects in the treatment of cancer. Anti-cancer effects are due to the virus ability: i) to replicate in and kill tumor cells, leading finally to their selective elimination; and ii) to induce the stimulation of antitumor activities in immune cells. NDV does not harm normal cells and has a high safety profile. In this study, we first report a direct correlation between the degree of cell resistance to NDV infection and the cellular expression of the retinoic acid-inducible gene I (RIG-I) which is a cytosolic viral RNA receptor. RIG-I plays an important role in the recognition of and response to infection by RNA viruses. We also demonstrate that impairment of the interferon (IFN) pathway through deletion of the receptor for type I IFN (IFNR1) in primary macrophages leads to NDV replication. In tumor cells, addition of exogenous IFN- $\alpha 4$ is shown to lead to tumor growth reduction and inhibition of viral replication. Finally, increase of the RIG-I concentration of tumor cells via plasmid transfection is shown to be associated with a stronger resistance to NDV infection. These findings shed new light on the crucial role played by the cytosolic receptor RIG-I and the plasma membrane receptor IFNR1 as key molecules to protect cells against infection by NDV.
\end{abstract}

\section{Introduction}

The use of oncolytic viruses (OVs) to treat cancer is not a new concept. Over the past decade, hundreds of patients have been treated in phase I and II trials with a diverse assortment of

Correspondence to: Professor Volker Schirrmacher, German Cancer Research Center (DKFZ), Im Neuenheimer Feld 280, D-69120 Heidelberg, Germany

E-mail: v.schirrmacher@dkfz.de

Key words: oncolytic virus, Newcastle disease virus, virus replication, innate cellular response, RIG-I, normal versus tumor cells, cellular toxicity, safety, cellular antiviral response, type I interferon, tumor selectivity
OVs (reviewed in ref. 1). The basis of the use of such biological agents is their capacity to destroy neoplastic cells while sparing untransformed cells. One advantage of OVs compared to other anticancer drugs is their self-replicating capacity in cancer tissue allowing local self-amplification of the biological agent. Newcastle disease virus (NDV) strains have a long history as oncolytic agents (2). NDV is a member of the Avulavirus genus in the Paramyxoviridae family. It infects a number of avian species (3). It is an enveloped virus of $150-300 \mathrm{~nm}$ size containing a non-segmented, single stranded RNA genome of negative orientation and of $15 \mathrm{~kb}$ size. Six viral genes code for the nucleocapsid protein (NP), the phosphoprotein (P), the matrix protein $(\mathrm{M})$, the fusion protein $(\mathrm{F})$, the hemagglutinin-neuraminidase $(\mathrm{HN})$ and the large protein (L) (4). After HN-mediated binding, the $F$ protein induces the fusion of the viral envelope with the host cell membrane and the nucleocapsid enters the cytosol, where the whole viral replication takes place until virus is released from the cells by budding. Over the last years, reverse genetics has allowed to modify the RNA genome of this virus. Following such procedures, we produced the following NDV recombinant viruses with transgenes coding for enhanced green fluorescent protein (EGFP) $(5,6)$, interleukin-2 (IL-2) $(7,8)$ or granulocyte macrophage colony stimulating factor (GM-CSF) (9).

Studies over the last decade have shown that initiation of innate immune responses is achieved through recognition of invading viruses by pattern recognition receptors (PRR) that specifically recognize pathogen-associated molecular patterns (PAMPs) and damage-associated molecular patterns (DAMPs) resulting from the viral infection. Virus-derived nucleic acids are considered major PAMPs that activate various PRRs, including members of the membrane-bound Toll-like receptor (TLR) family, TLR-3, -7 and -9, and the recently identified cytoplasmic RIG-I-like receptors (RLRs), including the retinoic acid inducible gene I RIG-I (10). RIG-I has the structural combination of an $\mathrm{N}$-terminal caspase recruitment domain (CARD) and a C-terminal DExD/H RNA helicase domain (11). In contrast to TLR3, which is expressed restrictedly to a specific cell type and binds to extracellular and endosomal dsRNA, RIG-I is expressed ubiquitously in different tissues. It is localized in the cytosol and recognizes 5'-triphosphate RNA (pppRNA) generated by viral RNA polymerases. Of note, pppRNA is also generated physiologically in the nucleus, but 
due to processing (such as splicing and addition of a 5' cap), it loses its RIG-I ligand activity before it is released to the cytosol $(12,13)$. This allows to recognize RNA motifs associated with danger signals such as the intracellular double-stranded RNA (dsRNA). RIG-I is a PRR recognizing foreign RNA and signs of replication of RNA viruses such as NDV. RIG-I appears important since mouse fibroblasts and dendritic cells from $\mathrm{RIG}^{-\mathrm{I}^{-/}}$mice fail to generate IFN- $\beta$ upon RNA virus infection when compared to their wt counterparts (14). It is also essential for NDV-induced IFN production in most cells, but it is dispensable for virus-induced interferon (IFN) production by pDCs (15). Following recognition of viral RNAs, RIG-I/MDA5 (melanoma-differentiation-associated gene 5, also known as Ifih1 or Helicard) elicits signaling cascades via a caspase recruitment domain (CARD)-mediated interaction with the mitochondriaassociated adaptor MAVS, also known as CARDIF/IPS-1/VISA (10). This molecule in turn interacts with the TNFR-associated death domain (TRADD) protein which is linked with death receptor (16). At this level, the signaling cascades diverge due to specific interactions either with the Fas-associated protein with the 2 adaptors [death domain (FADD) and receptor-interacting protein 1 (RIP1)] or with the E3 ubiquitin ligase TNF receptorassociated factor 3 (TRAF3) and the adaptor protein TANK, to elicit activation of the NF- $\kappa \mathrm{B}$ and IRF-3 transcription factors, respectively (16). Activation of the ubiquitously expressed IRF-3 transcription factor is central to the development of an antiviral state since it has the capacity to directly regulate a subset of these antiviral genes, including IFIT1 (interferoninduced protein with tetratricopeptide repeats 1 ), which encodes for the ISG56 translation regulator, thereby establishing an early IFN-independent antiviral response (17). Type I IFNs, which are central for the first line of defense against many viral infections and mediate antitumor activities of NDV, are a family of cytokines comprising more than 12 IFN- $\alpha$ and 1 IFN- $\beta$ species. All of these are important for the defense against viral infections (18). The rapid and robust expression of type I IFN genes is a prerequisite for the induction of numerous antiviral proteins (IFN-stimulated genes or ISG) that modulate protein synthesis, growth arrest and apoptosis (19). In addition to their anti-viral functions, IFNs also have pleiotropic antitumor activity (20).

In this study, we analyzed the viral transcription of NDV in tumor cells and normal cells. We report the existence of a direct correlation between the degree of cell resistance to NDV infection and the cellular expression in RIG-I which is measured before and after NDV infection. Furthermore, knocking out the type I interferon receptor in primary MEF cells allowed NDV replication, showing that the interferon feedback loop machinery is important. Addition of exogenous type I IFN reduced NDV replication within tumor cells but it did not show any increase in RIG-I expression, suggesting deficiencies in the interferon signaling in tumor cells. Last but not least, transfer of RIG-I expression plasmids into tumor cells led to decreased viral transcription upon NDV infection, highlighting an important role of RIG-I in restricting cellular replication of NDV.

\section{Materials and methods}

Mice and cells. C57BL/6 and Balb/c wt mice were purchased from Charles River Laboratories (Sulzfeld, Germany). The IFNAR $^{-1-}$ mice on a $\mathrm{C} 57 \mathrm{Bl} / 6$ background were kindly given by Dr R. Zawatsky (Division of Viral Transformation Mechanisms, DKFZ, Heidelberg, Germany). Murine spleen cells were prepared extemporaneously from mice. Murine macrophages were generated by growing bone marrow cells flushed from femora obtained from mice. The freshly harvested cells were then seeded $\left(4 \times 10^{6}\right.$ cells per petri dish having a $10-\mathrm{cm}$ diameter) and were grown for 8-12 days in RPMI-1640 medium supplemented with $10 \%$ FCS and $20 \%$ v/v M-CSF containing supernatant from a dense culture of L929 cells monolayer. After 10 days, the adherent cells were recovered from the cell culture plates and the macrophage phenotype determined by flow cytometry after staining with a monoclonal anti-F4/80 antibody (Cedarlane Laboratories, Burlington, Canada). Expression of the F4/80 macrophage marker was found in $>80-90 \%$ of the cells. The tumor cell lines: CT26p, CT26wt, the reticulum cell sarcoma (strain BALB/c) J774A.1 (here called J774), the macrophage-derived Abelson murine leukemia virus-induced tumor (strain BALB/c) RAW 264.7 (here called RAW), the breast adenocarcinoma (strain DBA/2) DA3, ESb, B16 and the human fibroblast cell line L929 (all obtained from the DKFZ tumor bank) were kept in culture in RPMI with 5\% FCS and glutamine, penicillin and streptomycin as additives.

Viruses and infections. The non-lytic, avirulent lentogenic NDV Ulster 2C (named NDV Ulster in this study), was provided originally by Dr P.H. Russel (Department of Microbiology and Parasitology, Royal Veterinary College, University of London, UK). Recombinant NDV expressing expressing the jellyfish enhanced green fluorescent protein (EGFP), named NDFLEGFP, was generated from a cDNA clone of the lentogenic NDV strain LaSota and was kindly provided by Dr B. Peeters (Division of Infectious Diseases, Animal Sciences Group, Wageningen UR, AB Lelystad, The Netherlands). Both NDV viruses were propagated in embryonated chicken eggs, harvested from the allantoic fluid, purified by ultracentrifugation and characterized as described (21). Quantification was performed by hemagglutination assay. The smallest virus concentration leading to visible agglutination of sheep erythrocytes is defined as 1 hemagglutination unit (HU) (21). For infection, cell suspensions were washed twice with FCS-free medium and $1 \times 10^{6}$ cells were incubated with $10 \mathrm{HU}$ of NDV in a final volume of $100 \mu \mathrm{l}$ for $1 \mathrm{~h}$ at $37^{\circ} \mathrm{C}$ in a cell culture incubator. During the incubation, cells were shaken every $15 \mathrm{~min}$. The cells were then washed twice and cultured further in $2 \mathrm{ml}$ medium with $10 \%$ FCS in 12-well plates.

Antibodies and flow cytometry. The following antibodies were used during this study: anti-HN (HN.B, mouse IgG2a, obtained from Dr R.M. Iorio (USA), goat F(ab')2 anti-mouse IgM, IgG, IgA $(\mathrm{H}+\mathrm{L})$ labeled with PE (Southern Biotech, Birmingham, USA). Monoclonal anti-F4/80-PE antibodies were obtained from Cedarlane Labs, Burlington, Ontario, Canada. Mouse rIFN- $\alpha 4$ was obtained from the culture medium of Chinese hamster ovary $(\mathrm{CHO})$ cells transfected with an expression vector encoding mouse IFN- $\alpha 4$ cDNA which was provided by Dr Rainer Zawatzky (DKFZ, Heidelberg, Germany). For flow cytometry analysis, between $1 \times 10^{5}$ and $1 \times 10^{6}$ cells were given in a FACS tube and centrifuged $\left(250 \mathrm{~g}, 3 \mathrm{~min}, 4^{\circ} \mathrm{C}\right)$ and incubated for $30 \mathrm{~min}$ on ice with the primary antibody in $50 \mu 1$. The primary antibodies were diluted 1:100 in FACS buffer. 
The samples were then washed twice with $300 \mu 1$ FACS buffer $\left(250 \mathrm{~g}, 3 \mathrm{~min}, 4^{\circ} \mathrm{C}\right.$ ) before they were resuspended in $50 \mu 1$ with the secondary fluorescently labeled antibody diluted 1:100 in FACS buffer. FACS buffer is 1X PBS with 5\% (v/v) FCS solution and $0.1 \%(\mathrm{w} / \mathrm{v})$ sodium azide. The propidium iodide solution $(1 \mu \mathrm{g} / \mu \mathrm{l})$ was made in PBS at a final concentration of $1 \mathrm{ng} / \mu \mathrm{l}$. All FACS data were analyzed with the WinMDI 2.8 software (Joe Trotter, The Scripps Research Institute, CA, USA).

$R N A$ isolation, $c D N A$ preparation and quantitative real-time PCR ( $q P C R)$. Cells were washed in PBS and cell pellets were either shock-frozen in liquid nitrogen for storage at $-80^{\circ} \mathrm{C}$ or immediately used for RNA isolation. Cell pellets were either frozen at $-80^{\circ} \mathrm{C}$ after removal of the medium or used immediately for RNA preparation. Cells were disrupted using a rotor-stator homogeniser. Total RNA was extracted using the RNeasy mini kit (Qiagen, Hilden, Germany) according to the manufacturer's instructions. Total RNA (500 ng) was then reversely transcribed into cDNA using Oligo(dT)12-18 primers and the SuperScript ${ }^{\mathrm{TM}}$ II Reverse Transcriptase (both Invitrogen, Karlsruhe, Germany) according to the manufacturer's instructions. For each assay, $500 \mathrm{ng}$ total RNA per sample was reversely transcribed using oligo(dT)12-18 primers and the generated cDNA was used in subsequent PCR reactions. For the real-time PCR, primers which bound just once in the genome when tested in a database search (BLAST, NCBI) were designed. The primers were optimized to show a melting curve with one peak only and to have approximately the same amplification efficiency as the gene used for normalisation. The master mix was prepared as recommended by the manufacturer (Eurogentec, Seraing, Belgium) and the concentrations for the primers and for $\mathrm{MgCl}_{2}$ were adjusted. Quantitative PCR was carried out using a GeneAmp ${ }^{\circledR} 5700$ Sequence Detection System (Applied Biosystems, Weiterstadt, Germany). After an initial incubation of $25 \mu \mathrm{l}$ reaction mix (Eurogentec, Seraing, Belgium) for $10 \mathrm{~min}$ at $95^{\circ} \mathrm{C}$, amplification was performed for 45 cycles consisting of a $15 \mathrm{sec}$ at $95^{\circ} \mathrm{C}$ and a $1 \mathrm{~min}$ at $60^{\circ} \mathrm{C}$. The expression of the target genes was normalised with the respective housekeeping gene after it had been ensured that the amplification efficiency was the same. For the viral $\mathbf{M}$ gene, the IFN- $\beta$ and $\beta$-actin detection, a fluorescent oligonucleotide probe system was used, while the other genes were detected with SYBR green. The sequences of the primers and the probes are listed (22).

Plasmids and transfection. Human RIG-I wild-type and human RIG-I IKA-mutated (11) were kindly provided by Dr Takashi Fujita, Tokyo Metropolitan Organization for Medical Research, Tokyo, Japan. To transfect mammalian cells with plasmid DNA, the cationic polymer transfection reagent jetPEI ${ }^{\mathrm{TM}}$ was used according to the manufacturer's instructions (PolyPlus, www. polyplustransfection.com, In vitro Transfection Protocol, Ref: CPT 101, Version H). The transfections were carried out on a 12-well plates.

Statistical analyses. All analyses were done with the GraphPad Prism 4.0 software (GraphPad Software, San Diego, CA, USA). $\mathrm{P}$-values were calculated with a two-sided Student's t-test.

\section{Results}

Normal cells in contrast to tumor cells show a strong resistance to replication of $N D V$. To analyze virus replication in normal and tumor cells, primary murine spleen cells and murine mammary adenocarcinoma DA3 cells were infected with the NDV strain Ulster. RNA was extracted at different time points after infection and the amount of viral $\mathrm{M}$ gene transcripts was determined by quantitative real-time PCR (qPCR). Spleen cells showed a low level of expression of viral M RNA (Fig. 1). In contrast, DA3 cells showed a strong increase in $\mathrm{M}$ gene expression between 4 and $8 \mathrm{~h}$ after infection and reached the maximum at $24 \mathrm{~h}$ postinfection (Fig. 1A). The maximum $\mathrm{M}$ gene expression in DA3 cells was $\sim 5000$ times higher than the one observed in spleen cells. Spleen cells appeared highly resistant to NDV infection, even when infected with high doses of NDV (data not shown). In order to rule out an effect of cell type in the resistance to NDV infection, we compared murine primary macrophages to two murine macrophage derived tumor cell lines, J774 and RAW. We used for this purpose a recombinant lentogenic NDV strain NDFL-EGFP, into which a marker transgene coding for EGFP has been inserted. Twenty-four hours after infection, the RAW cells showed a high EGFP signal, the J774 cells a lower signal while the normal macrophages expressed only a marginal signal (Fig. 1B). Since the differences observed in the expression of the $\mathrm{M}$ gene or of the EGFP protein, which were observed after NDV infection, could be due to differences in the ability of the cells to bind NDV, the cell binding capacity of NDV to normal cells and tumor cells was analyzed. The virus bound to cell surfaces was detected one hour after infection and washing by staining with a $\mathrm{HN}$-specific mAb and analysis by flow cytometry. The different cells used in infection assays showed similar binding of viral particles (data not shown). In order to further substantiate our observations, a certain number of normal and tumor cell types were infected with NDV Ulster and the M gene expression was measured with qPCR one day after infection. Fig. 1C shows that the $\mathrm{M}$ gene expression was higher in all tumor cells tested in comparison to normal cells. The difference was, however, not statistically significant because of the high variability of $\mathrm{M}$ gene expression in the tumor cells. A group with relatively low $\mathrm{M}$ gene expression comprised the CT26p [23 relative expression units (REU)], J774A.1 (64 REU) and RAW 264.7 cells (3749 REU). A medium expression of the $M$ gene was observed by DA3 (20821 REU), CT26wt (21063 REU) and B16 cells (19973 REU), and a high expression by the ESb cells (44529 REU). The M gene level in normal cells was always lower than 31 REU. The results demonstrate that normal cells are much more resistant to NDV infection than tumor cells.

The resistance to NDV replication in normal cells appears to be inversely related to RIG-I gene expression. In order to elucidate a possible reason for the stronger resistance of normal cells to NDV infection in comparison to tumor cells, we tested several normal and tumor cells for expression of RIG-I by quantitative PCR (qPCR). Higher expression of RIG-I was observed in normal versus tumor cells before (Fig. 2Aa, $\mathrm{p}=0.0109$ ) and after (Fig. 2Ab, p=0.0009) NDV infection. We then correlated the RIG-I expression to the $M$ gene expression. Fig. 2B shows a correlation between the basal (a) and the inducible (b) RIG-I 

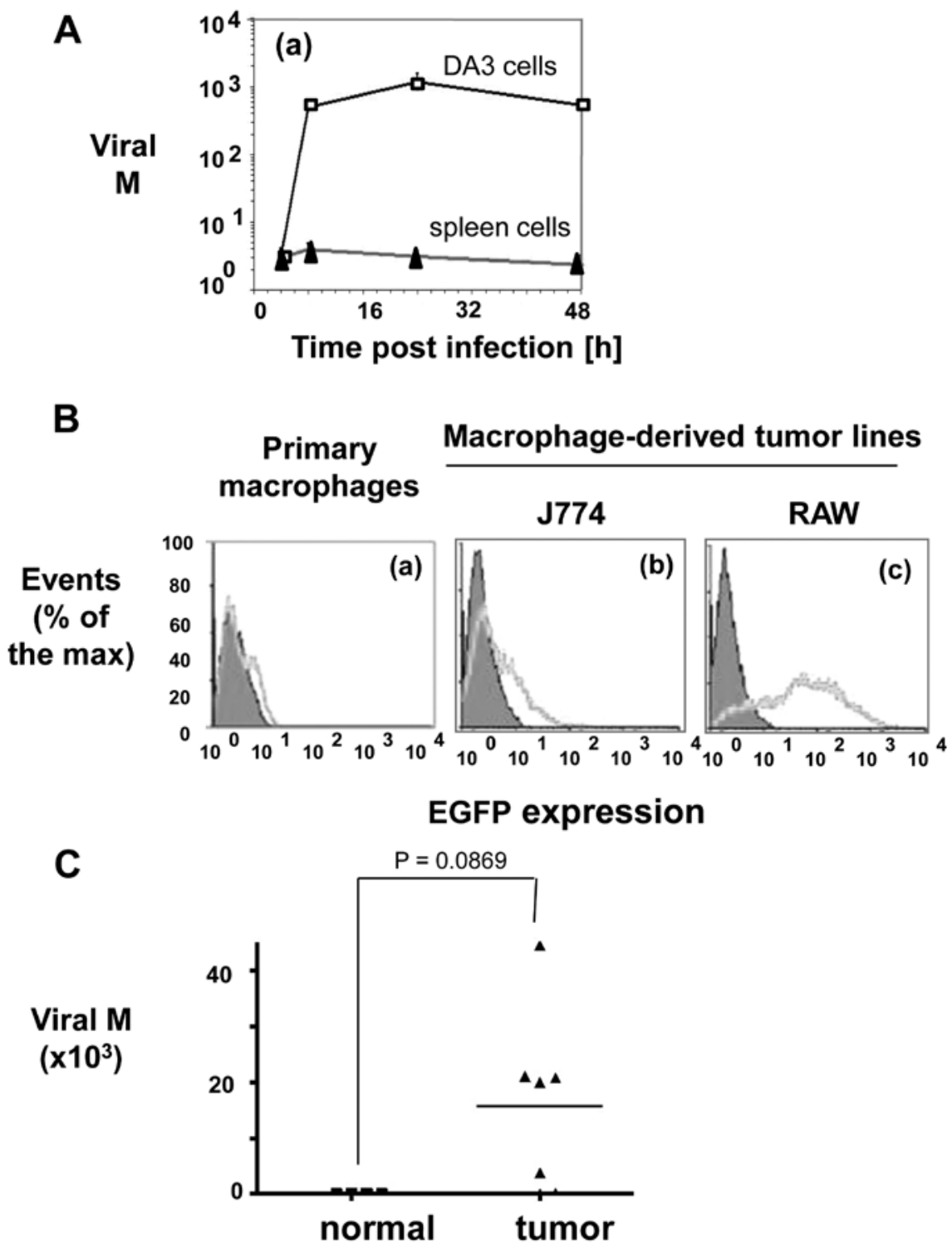

Figure 1. NDV replicates in tumor cells but not in normal cells. (A) NDV replication in murine spleen cells and DA3 tumor cells in vitro. Murine DA3 mammary adenocarcinoma cells and primary spleen cells, which were freshly isolated from DBA/ 2 mice, were infected with 10 HU NDV Ulster per $10^{6}$ cells as described in Materials and methods. At 0, 4, 8, 24 and $48 \mathrm{~h}$ post-infection, total RNA was prepared and, after reverse transcription, the amount of viral M gene cDNA was quantified by qPCR using the comparative CT method and $\beta$-actin for normalisation. At $0 \mathrm{~h}$, no $\mathrm{M}$ gene could be detected (data not shown). The results are represented as the mean of three measurements and bars indicate standard deviation. (B) EGFP expression after infection of macrophages, J774 and RAW tumor cells with the virus NDFL-EGFP. Bone marrow-derived primary macrophages from BALB/c mice (a), J774 (b) and RAW (c) tumor cells were infected with $10 \mathrm{HU}$ per $10^{6}$ cells of NDFL-EGFP. Twenty-four hours after infection, EGFP expression within the cells was determined by flow cytometry as described in Materias and methods. (C) Viral M gene expression in tumor cells and primary cell types after NDV infection. Several primary cell types (spleen cells and macrophages from BALB/c, C57BL/6 and DBA/2 mice) and tumor cell lines (CT26p, CT26wt, J774A.1, RAW 264.7, DA3, Esb and B16) were infected with $10 \mathrm{HU}$ of NDV (either NDV Ulster or NDFL-EGFP) per $10^{6}$ cells. Total RNA was extracted $24 \mathrm{~h}$ after infection. The expression level of the NDV M gene was determined by qPCR with the comparative CT method using $\beta$-actin for normalisation. P-value was calculated using an unpaired Student's t-test.

expression levels with the $\mathrm{M}$ gene expression when both parameters were plotted at a double logarithmic plot.

Impairment of the interferon signaling pathway allows NDV replication in mouse embryonic fibroblasts. In order to study the role of the interferon feedback-loop pathway in the resistance to NDV infection, mouse embryonic fibroblast (MEF) cells, either derived from wild-type (wt) or from $\alpha$ chain of the type 1 interferon receptor (IFNAR) knock-out (KO) mice, were used in infection experiments using different conditions. In some assays, the cells were treated with anti-IFN $\alpha / \beta$ for 7 days before NDV infection (group anti-IFN $\alpha / \beta$ ) and a part of these cells were also incubated with IFN $\alpha 4$ for $4 \mathrm{~h}$ just before NDV infection. The first parameter that was tested was the EGFP or $M$ gene expression after infection with NDFL-EGFP or NDV Ulster, respectively. Fig. 3A shows that the MEF wt cells had a much lower EGFP expression (a) and M gene expression (b) after NDV infection than the IFNAR1 KO counterpart cells. The EGFP and $M$ gene signals in the wt cells were not influenced by anti-IFN- $\alpha / \beta$ antibody pretreatment. However, MEF cells pretreated with IFN- $\alpha 4$ showed a marked reduction of $\mathrm{M}$ gene expression. In the IFNAR KO MEF cells, no pretreat- 
A

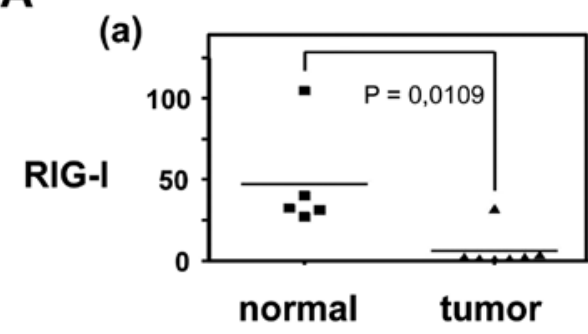

B

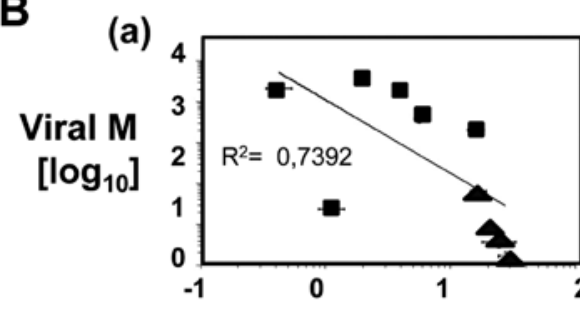

(b)



\section{RIG-I [ $\left.\log _{10}\right]$}

Figure 2. Relationship between RIG-I expression and viral replication. (A) Comparison of the RIG-I expression in primary and tumor cells before (a) and after (b) NDV infection. The RIG-I expression level was defined by qPCR in several primary and tumor cell types before and $24 \mathrm{~h}$ after infection with $10 \mathrm{HU}$ NDV Ulster or NDFL-EGFP. The gene expression was determined with the comparative CT method using the acidic ribosomal phosphoprotein PO for normalisation. The basal (a) and induced (b) RIG-I expression of several primary cells ( $\mathbf{m}$, macrophages from BALB/c, C57BL/6 and DBA/2 mice, lung tissue, liver tissue) and tumor cell types (^, CT26p, CT26wt, J774A.1, RAW 264.7, DA3, Esb and B16) is shown. P-value was calculated using an unpaired Student's t-test. (B) Inverse correlation between levels of RIG-I and viral M transcripts. The basal (a) and NDV induced (b) RIG-I levels in primary and tumor cells are plotted against the number of viral M transcripts which was determined $24 \mathrm{~h}$ after NDV infection. Bars indicate standard deviation. The correlation coefficient $\left(\mathrm{R}^{2}\right)$ was calculated with the mean of the expression values.

ment changed the EGFP or M gene signal (Fig. 3Ab). Upon infection with NDFL-EGFP, the IFNAR KO cells (Fig. 3Aa revealed two populations, one with a low and one with an 100 times stronger signal (10 versus 1000 relative fluorescence units). The low expressing cells appear similar to the wt cells. The IFNR1 appears to be a crucial factor for NDV resistance of the high expressing cells.

One explanation for the clear difference in resistance to NDV infection between wt and IFNAR KO MEF cells could be a difference in the expression of genes related to the antiviral interferon response. Therefore, the expression of three genes (RIG-I, IRF3 and IFN- $\beta$ ), which are important for the establishment of an interferon response, was determined by qPCR after NDV infection. Fig. 3B shows that the basal expression of RIG-I, IRF-7 and IFN- $\beta$ was moderately higher in the IFNAR $\mathrm{KO}$ than in the wt MEF cells. IFN- $\alpha 4$ pretreatment increased the basal gene expression only in the wt cells and it reduced the gene expression after NDV infection solely in these cells. The increase in basal gene expression by IFN- $\alpha 4$ pretreatment was highest for IRF-7 (7-fold) and lower for RIG-I (3-fold) and IFN- $\beta$ (2-fold). After infection with NDV Ulster, the IFN- $\beta$ expression in the IFNAR KO cells was 10-15 times higher and the RIG-I expression 3 times higher than in the wt cells. The IRF-7 expression after infection was comparable in both MEF cell types. IFN- $\alpha 4$ pretreatment increased basal gene expression and led to a reduced expression level after NDV infection only in wt and not in IFNAR KO cells, in accord with the assumption that IFN- $\alpha 4$ signals exclusively through the IFNAR. The stronger expression of IFN- $\beta$ and RIG-I after NDV infection of IFNAR KO cells could be explained by more viral danger signals produced due to the better NDV replication in these cells.

The interferon signaling pathway appears impaired in tumor cells. Important factors for cellular resistance to virus infection are their production and secretion of interferon and their responsiveness to external interferon. In order to study the effects of type I interferon on virus replication, J774 and RAW cells were infected with NDV and then incubated with or without IFN- $\alpha 4$. Their growth was then analyzed by counting the cell numbers in both conditions. Fig. 4A shows a reduction of cell growth in the presence of IFN. The samples were also taken at different time-points after NDV infection for RNA extraction and analyzed for $\mathrm{M}$ gene expression by qPCR. Fig. 4B shows distinct differences in $\mathrm{M}$ gene expression for the three cell types. For the macrophages, the $\mathrm{M}$ gene level remained low while for the other two cell types the $\mathrm{M}$ gene level increased with similar kinetics to reach a plateau at $12 \mathrm{~h}$ after infection. When the peak of $\mathrm{M}$ gene expression of the macrophages (11 REU) was compared to the highest expression of the two tumor cell lines, we could estimate the increase in susceptibility to NDV infection by the tumor cells. The J774 cells were 20-50 times more susceptible than the macrophages and the RAW cells 300-400 times more. It was also of interest to compare infection kinetics of a lentogenic strain such as Ulster with a more virulent strain such as NDV Italian which is velogenic. Overall, the $\mathrm{M}$ gene expression was 2-5 times higher when the three cell types were infected with NDV Italien compared to NDV Ulster (data not shown). But the ranking order of the cell types with regard to sensitivity to infection by NDV remained the same 

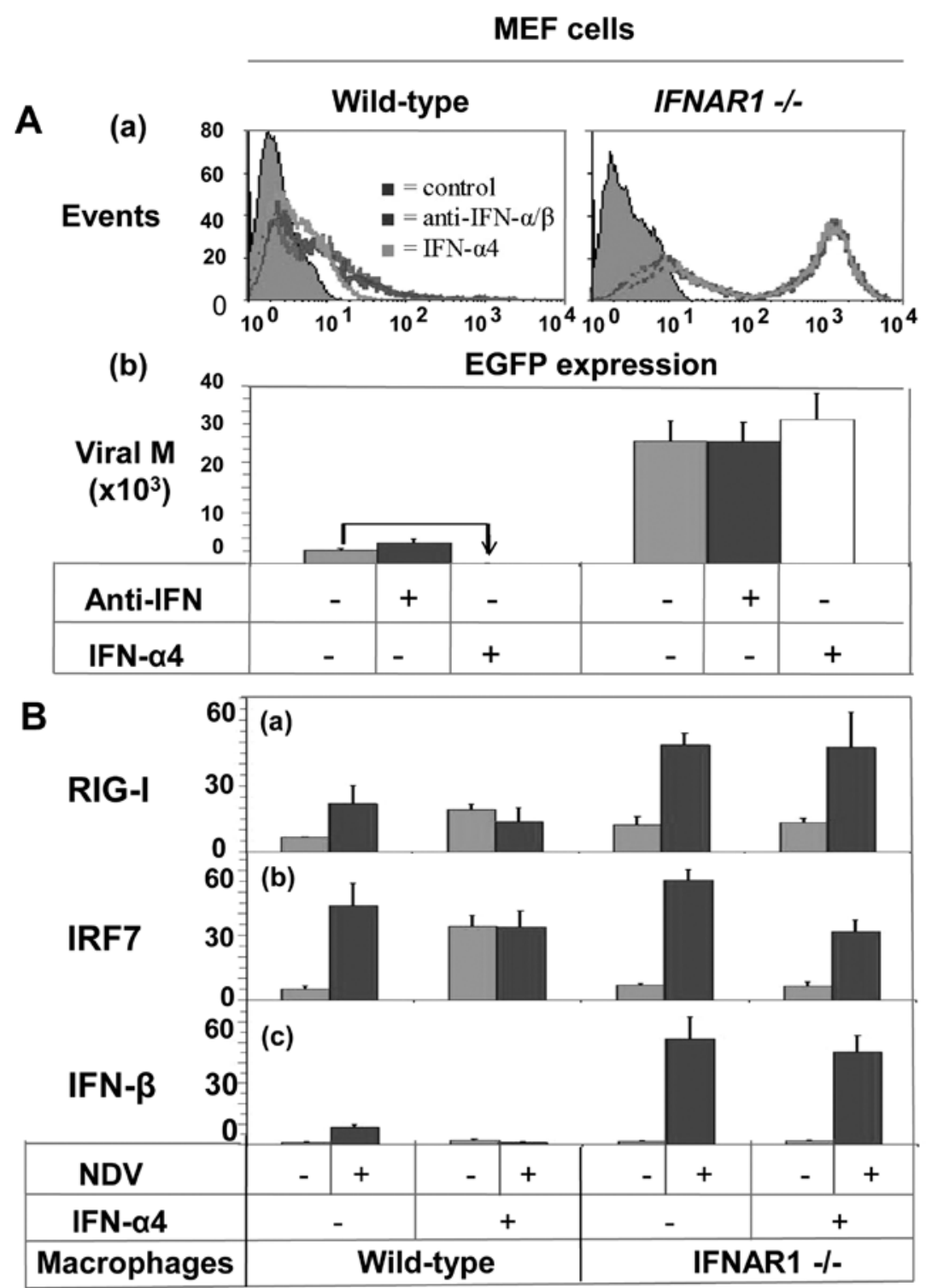

Figure 3. Importance of type I interferon receptor for cellular resistance to NDV infection. (A) Replication of NDV in IFNAR1 $1^{-/}$MEF cells in comparison to their wt counterparts. Wt and IFNAR ${ }^{-/}$mouse embryonic fibroblasts were grown for 7 days either in normal growth medium ( $\mathbf{m}$, control group) or in medium



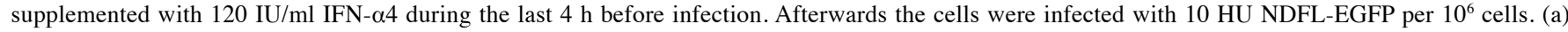
The EGFP expression was determined by flow cytometry $20 \mathrm{~h}$ later. (b) Twenty hours after infection total RNA was extracted and the expression level of the viral $\mathrm{M}$ transcripts was determined by qPCR with the comparative CT method using $\beta$-actin for normalisation. The results are presented as the mean of three measurements. The percentage of $\mathrm{M}$ gene expression blocking in comparison to the control is indicated. Bars indicate standard deviation within triplicates. (B) Interferon-related gene expression in wt or IFNAR ${ }^{-1}$ MEF cells after infection with NDV. From the extracted RNA in (A), the expression level of the RIG-I (a), the IRF-7 (b) and the IFN- $\beta$ (c) gene was determined by qPCR with the comparative CT method using the acidic ribosomal phosphoprotein PO for normalisation. The results of the different conditions, which were tested, are presented as the mean of three measurements. Bars indicate standard deviation.

(data not shown), the primary macrophages being resistant to NDV infection (Fig. 1B). Fig. 4B shows that in J774 tumor cells, IFN- $\alpha 4$ pre-treatment totally abolished $\mathrm{M}$ gene expression at any time-point. The $\mathrm{M}$ gene expression in the RAW cells was only partially suppressed by pre-treatment with IFN- $\alpha 4$. Eight to twenty hours after infection, the rate of $\mathrm{M}$ gene increase was similar for the pre-treated and for the control RAW cells. Thus, the interferon signaling pathway appeared to be impaired in the highly susceptible tumor line RAW in comparison to the low susceptible line $\mathbf{J} 744$.

Addition of exogenous IFNa4 can not restore RIG-I deficiency in tumor cells. We next analyzed the influence of IFN- $\alpha 4$ on the interferon-related gene expression. The expression of RIG-I, IRF-3, IRF-7 and IFN- $\beta$ was analysed with and without IFN- $\alpha 4$ pretreatment before and after NDV infection with real-time RT-PCR. Fig. 4Ca shows that the IFN- $\alpha 4$ pretreatment induced RIG-I expression in all three cell types. The induction was $\sim 5$-fold in macrophages, 2-fold in J774 cells and 3-fold in RAW cells. In all three cell types, NDV Ulster infection induced a stronger RIG-I expression than the pretreatment with IFN- $\alpha 4$. When pretreated with IFN- $\alpha 4$, macrophages displayed a higher RIG-I expression level after NDV infection than the two tumor cell lines. In addition the pretreated macrophages had a basal RIG-I expression level that was higher than that in the tumor cells after NDV infection. The induction of RIG-I was strongest 


\section{Primary macrophages \\ Macrophage-derived tumor lines}

A


Figure 4. Influence of exogenous IFN- $\alpha 4$ on tumor growth, virus replication and RIG-I expression. (A) Effect of NDV and exogenously added IFN- $\alpha 4$ on tumor cell growth. Primary macrophages $\left(7.5 \times 10^{5}\right)$ (a), J774 (b) and RAW (c) cells were infected with $10 \mathrm{HU}$ NDV Ulster per $10^{6}$ cells, seeded in 6-well plates and treated () or not (घ) with exogenously added IFN- $\alpha 4$ (at a final concentration of $120 \mathrm{IU} / \mathrm{ml}$ ). Subsequently at each time-point up to 4 days the number of living cells in one well was counted with a hemocytometer and an optical microscope. In order to stain dead cells trypan blue was used. (B) Effect of IFN- $\alpha 4$ pretreatment on NDV replication. Primary macrophages (a), J774 (b) and RAW tumor cells (c) (3x10 cells per well in 6-well plates) were grown in normal growth medium

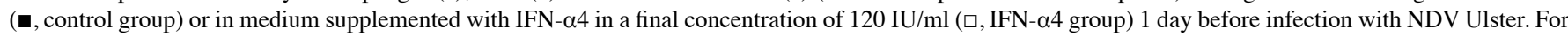
the infection $10 \mathrm{HU}$ per $10^{6}$ cells, NDV Ulster was used and total RNA was extracted at $0,4,8$ and $20 \mathrm{~h}$ after infection. After a reverse transcription the expression level of the NDV M gene was determined by qPCR with the comparative CT method using $\beta$-actin for normalisation. (C) Influence of IFN- $\alpha 4$ pretreatment on RIG-I (a), IRF-3 (b) and IRF-7 (c) expression in macrophages, J774 and RAW tumor cells after NDV infection. The level of RIG-I (a), IRF-3 (b) and IRF-7 (c) expression in macrophages, J774 and RAW tumor cells after NDV infection was determined by qPCR at $20 \mathrm{~h}$ post infection with the comparative CT method using the acidic ribosomal phosphoprotein PO for normalisation. The results are presented as the mean of three measurements. Bars indicate standard deviation.

in macrophages, lower in $\mathbf{J 7 7 4}$ cells and lowest in RAW cells. A difference between the macrophages and the two tumor cell lines with regard to IFN- $\alpha 4$ responsiveness was that, after NDV infection, the RIG-I expression level was higher in pretreated macrophages than in pretreated tumor cells. Fig. $4 \mathrm{Cb}$ shows that the IRF-3 response to NDV was not affected by IFN- $\alpha 4$. The IRF-3 expression level was almost the same in the pretreated and in the control cells. NDV Ulster infection only stimulated IRF-3 expression in macrophages and not in the two tumor cell lines. Fig. 4Cc shows that the IRF-7 response to NDV was reduced by IFN- $\alpha 4$ in all three cell types. The strongest effect was seen in macrophages ( $\sim 30$-fold). In J774 cells, the effect was $\sim 8$-fold and lowest in RAW cells ( 4-fold).

Transfection of RIG-I in tumor cells leads to reduced NDV replication. In order to investigate the functional significance of RIG-I during NDV infection, an expression plasmid coding for the human RIG-I was transfected into L929 fibroblasts. For control, the cells were transfected with the plasmid RIG-I IKA coding for the RIG-I protein with a mutation making it 


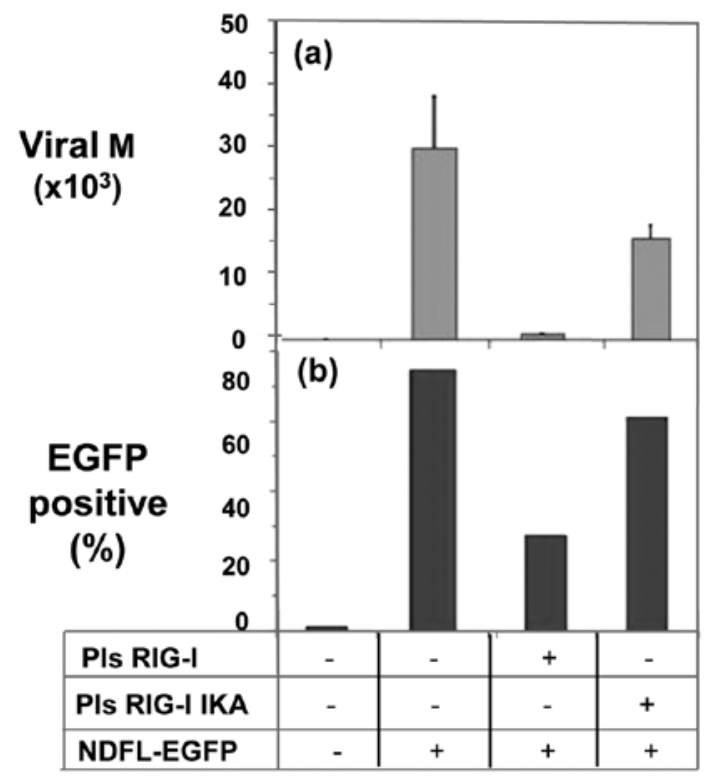

Figure 5. Increasing RIG-I expression via plasmid transfection leads to reduction of NDV replication. L929 murine fibroblast cells were transfected with expression plasmids coding for the wild-type human RIG-I (pRIG-I wt) or for a mutated, non-functional RIG-I IKA (pRIG-I IKA). jetPEI ${ }^{\mathrm{TM}}$ was used as transfection reagent as described in Materials and methods. Twenty-four hours later, the cells were infected with NDFL-EGFP. (a) Total RNA was extracted $24 \mathrm{~h}$ after infection and the expression level of the NDV M gene was determined by qPCR with the comparative $\mathrm{CT}$ method using $\beta$-actin for normalisation. The results are presented as the mean of three measurements. Bars indicate standard deviation. (b) Twenty-four hours after NDFL-EGFP infection, the EGFP expression was determined by flow cytometry and the \% of EGFP positive cells was calculated for each condition.

non-functional. Twenty-four hours after transfection, the cells were infected with NDFL-EGFP. One day later, the quantity of viral $\mathrm{M}$ transcripts was determined by qPCR. In parallel, the cells were analyzed by flow cytometry for EGFP fluorescence. A clear difference in the expression of NDV genes between the L929 cells transfected with the 2 different RIG-I plasmids was observed (Fig. 5). M gene expression was clearly lower in RIG-I than in RIG-I IKA transfected cells [222 and 16679 REU, respectively (Fig. 5a)]. The cells transfected with RIG-I showed a lower EGFP expression (28\%) than the RIG-I IKA transfected cells $(62 \%)$ (Fig. 5b). We conclude that functional RIG-I molecules can increase resistance of tumor cells to NDV infection.

\section{Discussion}

The aim of this study was to examine a possible relation between the resistance of cells to infection by NDV and the cellular expression of receptor molecules for recognition of: i) viral RNA (e.g., RIG-I); and ii) external type I IFN (e.g., IFNR1). We observed with a panel of cells that normal cells, in contrast to tumor cells, show resistance to NDV infection, thus corroborating previous findings (23-25). This resistance of normal cells was associated with a higher basal expression level of RIG-I. It was also observed that, upon NDV infection, normal cells reacted with the induction of a higher number of RIG-I transcripts than tumor cells. Furthermore, deletion of the receptor IFNR1 in MEF cells was demonstrated to brake resistance to NDV infec- tion, highlighting the importance of the interferon loop pathway for the control of viral replication. Addition of IFN- $\alpha 4$ to tumor cells, which were not resistant to NDV infection, induced a partial protection against viral replication. Similarly, increasing RIG-I expression in tumor cells via transfection was shown to lead to reduction of viral replication. These observations offer a scientific explanation for the high safety profile and low side effects of NDV in its use in human clinical trials.

RIG-I appears as a crucial molecule to start an antiviral response after virus infection. In this study, it was observed that the basal and inducible expression level of this gene can be correlated with cellular resistance to infection by NDV. Such correlation was corroborated by the fact that J774 tumor cells with an intermediate NDV resistance also showed intermediate levels of basal and inducible RIG-I helicase expression. All findings of this study show that RIG-I can affect cell resistance to NDV infection. They offer an explanation for the difference between normal and tumor cells with regard to NDV replication and also for the lack of toxicity of NDV in vivo. High RIG-I expression of normal cells might explain the high safety level of this virus in humans. The cytosolic receptor RIG-I enables recognition of foreign viral RNA and initiates a cellular anti-viral response that prevents virus replication. This is true at least for the response to infection by avian paramyxoviruses such as NDV. Part of the anti-viral response involves type I interferons which are induced and which establish an antiviral state in virally-infected and also in uninfected cells which express receptors for type I IFN (e.g., IFNR1). Contact of humans with NDV leads only to asymptomatic or very mild pathology such as conjunctivitis. It has to be noted that NDV is an avian virus which had no chance to develop in humans any immune evasion strategy. This aspect is very important for the safety issues associated with the clinical use of a virus. Other reasons why NDV shows a high safety profile are for example the fact that the RNA genome of NDV does not undergo recombination with DNA of host cells or of other viruses.

Many tumor cells have been reported to have acquired defects in the interferon response that might give them a growth and survival advantage (26-31). The tumor selectivity of NDV has been related to a limited interferon response in human fibrosarcoma cells (32) or in human MCF-7, BT-20 and Jurkat tumor cells (33). NDV shows a preference for replicating in and killing a variety of tumor cells but not normal cells (34). In contrast to genetically modified OVs such as adenovirus or herpes simplex virus, the tumor selectivity of NDV is inherent which makes it an attractive candidate for virotherapy (23-25). NDV has been reported to replicate up to 10,000 times better in cancer cells than in most normal cells $(21,29,34-36)$. The analysis of about a dozen normal and tumor cells revealed an apparent correlation between the expression of RIG-I and cellular resistance to NDV infection (Fig. 2). A correlation between two variables is not necessarily a proof of a causal relation, but, because the antiviral effects of RIG-I are known, a function correlation appears to exist. It can thus be concluded that defects in the basal or inducible expression of RIG-I make cells less resistant to NDV infection. This should be the case not only for tumor cells but also for embryonic stem cells which were shown to have only a weak response to cytoplasmic viral dsRNA (37).

Recently, negative regulators of RIG-I signaling have been identified, including the zinc finger protein A20 (38) and NLR 


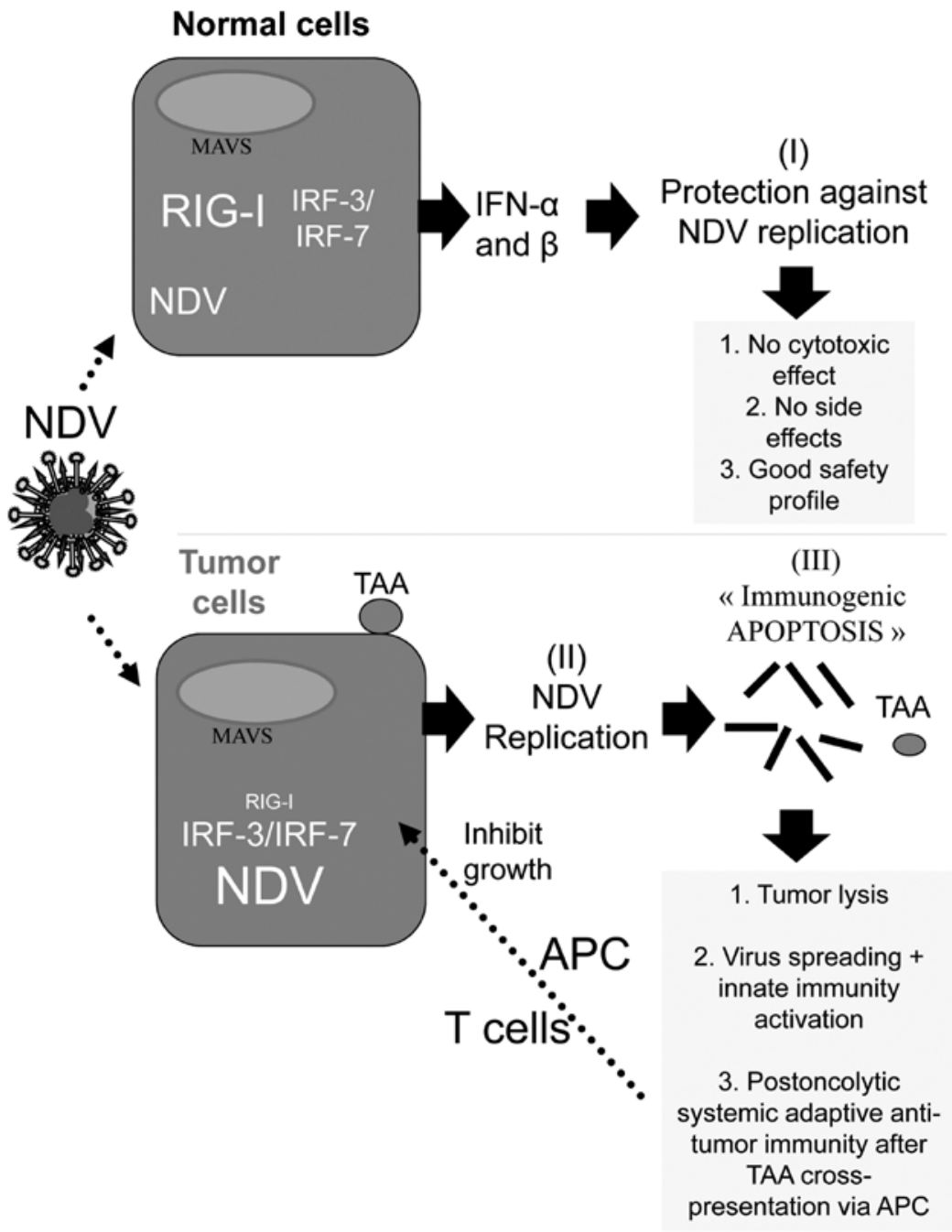

Figure 6. Scheme of the role of RIG-I for: i) resistance of normal cells to NDV replication; ii) susceptibility of tumor cells to NDV replication; and iii) immunogenicity of tumor cells (see Discussion for more details).

family member X1 [NLRX1 (39)]. However, the relevance of these negative regulators for physiological functions is not clear. They may play a role in the blocking of RIG-I signaling during oncogenesis and in tumor cells. Negative regulation of RIG-I signaling was reported by caspase 8 -mediated cleavage of the RIP-1 protein (40) and also by proteasomal degradation which is facilitated by the overexpression of caspase- 8 . Very interestingly, Su et al (41) demonstrated that RIG-I expression is downregulated in cancer cells when compared to normal cells. Its expression was primarily regulated by IRF-1. They observed also a positive correlation between expression of IRF-1 and RIG-I. In addition to its antiviral effects, IRF-1 has been shown previously to have tumor suppressor properties (42). In this context, we hypothesize that RIG-I may also function as a tumor suppressor. Its overexpression, which is induced in the host by NDV inoculation, may be a very powerful strategy for tumor cell treatment. Oncogenes could also interfere with RIG-I signaling. A recent study showed that oncogenic Ras promotes reovirus spread by suppressing IFN- $\beta$ production through negative regulation of RIG-I signals (43).

When different strains of NDV were used for the infection of normal and tumor cells, different levels of viral gene expression could be observed. Infection of macrophages with the velogenic
NDV strain Italien and to a lesser degree also with the mesogenic strain NDFLtag-EGFP generated a higher level of viral gene expression than the infection with the lentogenic strains Ulster and NDFL-EGFP (data not shown). As might have been expected, it seems that the more virulent an NDV strain is in birds, the better it can also replicate in mammalian cells. One explanation could be an increased ability of velogenic strains to limit the induction of antiviral responses. Interestingly, NDV Ulster induced stronger RIG-I gene expression than NDV Italien (data not shown). The proposed hypothesis is substantiated by the fact that, in macrophages, the induction of antiviral gene expression was generally lower for NDV Italien than for NDV Ulster (data not shown). The importance of RIG-I for virus recognition was shown to depend on the type of virus. For NDV, RIG-I has been reported to be more important than MDA5 (14). This might be due to the fact that most paramyxoviruses target MDA5, but not RIG-I with their V proteins (44). The functional significance of RIG-I in NDV infection was confirmed in transfection experiments with plasmids encoding functional or mutated non-functional RIG-I (Fig. 5).

The cell cycle status might also be important for giving more or less optimal conditions for viral replication. Low proliferation rates of normal cells contribute to their resistance to 
infection by NDV. In highly replicating tumor cells, the cellular machinery that is necessary for the replication of the cell may also serve virus replication. The murin embryonic fibroblasts MEF were susceptible to NDV infection although they are non-malignant cells. MEF cells are similar to an immortalised cell line that grows permanently in culture in contrast to, for instance, macrophages. MEF cells provide a better environment for NDV replication since much of the cellular machinery that is required for cell proliferation also allows for optimal virus replication. Another factor for susceptibility of MEF cells was apparently the downregulation of important interferon-related genes such as RIG-I, IRF-7 or IFN- $\beta$. The expression of these molecules might have decreased because cells with a low expression of interferon-related proteins are less sensitive to anti-growth effects of IFN and have therefore a higher proliferation rate in in vitro cell culture. Nevertheless, when IFNAR KO MEF cells were compared with the wt cells, there was a strong increase in NDV replication and a complete abrogation of the responsiveness to IFN- $\alpha 4$. Interestingly, the knock-out of the receptor IFNAR had almost no influence on the expression of the three interferon-related genes RIG-I, IRF-7 and IFN- $\beta$ (Fig. 3Bc), possibly because these genes were already expressed at a relatively low level in the wt MEF cells. In mice lacking a functional IFNR1, tumor development and ensuing mortality were enhanced, indicating that endogenous IFN- $\alpha / \beta$ production is a mediator of natural immunity against tumor development (45). The IFNR1 was found to be expressed on epithelial tumors but was missing in lymphomas, sarcomas and endocrine tumors (46), suggesting an involvement of this receptor in tumorigenesis of the latter cancer types. In addition, the transfection of tumor cells with a type I IFN receptor chain increased the rate of apoptosis and sensitivity to the antiproliferative effects of IFNs (31). In melanoma cells, defects in the IFNR1 JAK-STAT pathway led to cancer progression through loss of growth-restraining functions of IFN (26). The EGFP signal in the IFNAR1 KO MEF cells pointed to two populations with markedly different susceptibility to NDV infection, possibly representing two different stages in the cell cycle. According to this hypothesis, the highly NDV susceptible cells were proliferating and provided the virus with a high amount of the necessary cellular components for replication. The cells, which were more resistant to NDV infection, could be non-proliferating and would therefore be less suitable for NDV replication. The $\mathrm{M}$ gene expression kinetics revealed that the main increase in expression took place between 8-20 h after infection (Fig. 1A).

In this context, the role of mitochondria as a potential central platform of innate immune response is becoming appreciated. Recent data from the literature (47) indicate that mitochondria play a key role at the crossroad of bioenergetic metabolism, cell death signaling and induction of an innate immune response. The MAVS protein has been described as an adapter polypeptide which is present at the mitochondrial surface. After binding to the activated intracellular RIG-I receptor, MAVS activates IRFs and $\mathrm{NF}-\kappa \mathrm{B}$, thereby driving the production of type I interferon and proinflammatory cytokines, respectively (48). Danger signals (PAMPs, DAMPs) appear to be integrated by the mitochondria for the orchestration of the innate immune response and could be controlled by regulatory mechanisms such as mitophagy (49). The hypothesis suggesting that mitochondrial damages (such as inhibition of mitophagy, accumulation of damaged mitochondria) accumulate during oncogenesis is appealing in this regard. Increasing evidence suggests that RIG-I is more than a virus sensor and has a role in a series of biological events such as inflammation, proliferation and apoptosis (50). Aberrant immune signaling by RLR has been implied in the development of autoimmune diseases (51). A recent study (52) shows that mimicking viral infection in ovarian cancer cells by targeting RIG-I in these cells $(12,53)$ can be used to enhance anti-tumor activities. Monocytes and monocyte-derived dendritic cells that engulfed such modified and apoptotic cancer cells matured and secreted proinflammatory chemokines as well as type I IFN. Whereas most forms of apoptosis are considered 'immunologically silent' and tolerogenic (54), cell death initiated by RIG-I agonists was highly immune stimulatory with apoptotic tumor cells not only secreting proinflammatory cytokines and chemokines but also inducing functional maturation and type I IFN secretion of APCs and upregulation of HLA class I at the surface of the targeted cells. This could shift the balance away from tumor-induced immune suppression toward enhancement of cytotoxic antitumor immune responses. Especially, the destruction of the bulk tumor mass by an oncolytic NDV virus might open a window for optimal post-oncolytic antitumor effects. In particular, type I IFN which is produced upon NDV replication can activate NK cells, enhance dendritic cell mediated cross-priming and antigen-specific $\mathrm{CD}^{+} \mathrm{T}$ cell responses leading to increased anti-tumor immunity.

From the data of this study, we conclude that NDV is a very suitable oncolytic agent for cancer treatment. It possesses a good balance of potencies that renders it harmless to normal cells (with the induction of a strong innate antiviral response) whereas harboring strong lytic activities in tumor cells (associated with induction of strong adaptive anti-tumor immune response activities). Recent data show that RIG-I activation through NDV leads to suppression of the inhibitory activities of Tregs (55). These two properties appear linked to the same key molecule which is RIG-I. Tumor cell killing and immunostimulation through the oncolytic properties of NDV may then synergize for optimal anticancer therapy. This can be used in a multimodal approach together with standard therapies to achieve additional or even synergistic effects.

\section{Acknowledgements}

This study was part of the PhD thesis of Holger Wilden under co-supervision by Professor Volker Schirrmacher and Dr Philippe Fournier. We are particularly grateful to Annette Arnold for excellent technical assistance. We thank IOZK (www.IOZK.de) for financial help for the page charges of this manuscript.

\section{References}

1. Eager RM and Nemunaitis J: Clinical development directions in oncolytic viral therapy. Cancer Gene Ther 18: 305-317, 2011.

2. Sinkovics JG and Horvath JC: Newcastle disease virus (NDV): brief history of its oncolytic strains. J Clin Virol 16: 1-15, 2000.

3. Alexander DJ: Newcastle disease virus - an avian paramyxovirus. In: Newcastle Disease. Kluwer Academic, Dordrecht, pp1-22, 1988.

4. Nagai Y, Hamaguchi M and Toyoda T: Molecular biology of Newcastle disease virus. Prog Vet Microbiol Immunol 5: 16-64, 1998. 
5. Al-Garib SO, Gielkens AL, Gruys E, Peeters BP and Koch G: Tissue tropism in the chicken embryo of non-virulent and virulent Newcastle diseases strains that express green fluorescence protein. Avian Pathol 32: 591-596, 2003.

6. Peeters BP, De Leeuw OS, Koch G and Gielkens AL: Rescue of Newcastle disease virus from cloned cDNA: evidence that cleavability of the fusion protein is a major determinant for virulence. J Virol 73: 5001-5009, 1999.

7. Janke M, Peeters B, Zhao H, De Leeuw O, Moorman R, Arnold A, Ziouta Y, Fournier P and Schirrmacher V: Activation of human $\mathrm{T}$ cells by a tumor vaccine infected with recombinant Newcastle disease virus producing IL-2. Int J Oncol 33: 823-832, 2008.

8. Zhao H, Janke M, Fournier P and Schirrmacher V: Recombinant Newcastle disease virus expressing human interleukin-2 serves as a potential candidate for tumor therapy. Virus Res 136: 75-80, 2008.

9. Janke M, Peeters B, De Leeuw O, Moorman R, Arnold A, Fournier P and Schirrmacher V: Recombinant Newcastle disease virus (NDV) with inserted gene coding for GM-CSF as a new vector for cancer immunogene therapy. Gene Ther 14: 1639-1649, 2007.

10. Kawai T and Akira S: Toll-like receptor and RIG-I-like receptor signaling. Ann NY Acad Sci 1143: 1-20, 2008.

11. Yoneyama M, Kikuchi M, Natsukawa T, Shinobu N, Imaizumi T, Miyagishi M, Taira K, Akira S and Fujita T: The RNA helicase RIG-I has an essential function in double-strand RNA-induced innate antiviral response. Nat Immunol 5: 730-737, 2004.

12. Hornung V,Ellegast J, Kim S, Brzózka K, Jung A, Kato H,Poeck H, Akira S, Conzelmann KK, Schlee M, Endres S and Hartmann G: 5'-Triphosphate RNA is the ligand for RIG-I. Science 314: 994-997, 2006.

13. Pichlmair A, Schulz O, Tan CP, Näslund TI, Liljeström $P$, Weber F and Reis e Sousa C: RIG-I-mediated antiviral responses to single-stranded RNA bearing 5'-phosphates. Science 314: 997-1001, 2006.

14. Kato H, Takeuchi O, Sato S, Yoneyama M, Yamamoto M, Matsui K, Uematsu S, Jung A, Kawai T, Ishii KJ, Yamaguchi O, Otsu K, Tsujimura T, Koh CS, Reis e Sousa C, Matsuura Y, Fujita T and Akira S: Differential roles of MDA5 and RIG-I helicases in the recognition of RNA viruses. Nature 441: 101-105, 2006.

15. Kato H, Sato S, Yoneyama M, Yamamoto M, Uematsu S, Matsui K, Tsujimura T, Takeda K, Fujita T, Takeuchi O and Akira S: Cell type specific involvement of RIG-I in antiviral response. Immunity 23 : 19-28, 2005.

16. Michallet MC, Meylan E, Ermolaeva MA, Vazquez J, Rebsamen M, Curran J, Poeck H, Bscheider M, Hartmann G, König M, Kalinke U, Pasparakis $\mathrm{M}$ and Tschopp $\mathrm{J}$ : TRADD protein is an essential component of the RIG-like helicase antiviral pathway. Immunity 28: 651-661, 2008 .

17. Grandvaux N, Servant MJ, ten Oever B, Sen GC, Balachandran S, Barber GN, Lin R and Hiscott J: Transcriptional profiling of interferon regulatory factor 3 target genes: direct involvement in the regulation of interferon-stimulated genes. J Virol 76: 5532-5539, 2002.

18. Biron CA and Sen GC: Interferons and other cytokines. In: Fundamental Virology. Knipe DM and Howley PM (eds) Lippincott Williams and Wilkins, Philadelphia, PA, pp321-351, 2001.

19. Sen GC and Peters GA: Viral stress-inducible genes. Adv Virus Res 70: 233-263, 2007.

20. Dunn GP, Koebel CM and Schreiber RD: Interferons, immunity and cancer immunoediting. Nat Rev Immunol 6: 836-848, 2006.

21. Schirrmacher V, Haas C, Bonifer R, Ahlert T, Gerhards R and Ertel C: Human tumor cell modification by virus infection: an efficient and safe way to produce cancer vaccine with pleiotropic immune stimulatory properties when using Newcastle disease virus. Gene Ther 6: 63-73, 1999.

22. Wilden H, Fournier P, Zawatzky R and Schirrmacher V: Expression of RIG-I, IRF3, IFN-beta and IRF7 determines resistance or susceptibility of cells to infection by Newcastle disease virus. Int J Oncol 34: 971-982, 2009.

23. Freeman AI, Zakay-Rones Z, Gomori JM, Linetsky E, Rasooly L, Greenbaum E, Rozenman-Yair S, Panet A, Libson E, Irving CS Galun E and Siegal T: Phase I/II trial of intravenous NDV-HUJ oncolytic virus in recurrent glioblastoma multiforme. Mol Ther 13 : 221-228, 2006

24. Csatary LK, Gosztonyi G, Szeberenyi J, Fabian Z, Liszka V, Bodey B and Csatary CM: MTH-68/H oncolytic viral treatment in human high-grade gliomas. J Neurooncol 67: 83-93, 2004
25. Lorence RM, Roberts MS, O'Neil JD, Groene WS, Miller JA, Mueller SN and Bamat MK: Phase 1 clinical experience using intravenous administration of PV701, an oncolytic Newcastle disease virus. Curr Cancer Drug Targets 7: 157-167, 2007.

26. Pansky A, Hildebrand P, Fasler-Kan E, Baselgia L, Ketterer S, Beglinger $\mathrm{C}$ and Heim MH: Defective Jak-STAT signal transduction pathway in melanoma cells resistant to growth inhibition by interferon-alpha. Int J Cancer 85: 720-725, 2000.

27. Paucker K, Cantell K and Henle W: Quantitative studies on viral interference in suspended L cells. III. Effect of interfering viruses and interferon on the growth rate of cells. Virology 17: 324-334, 1962

28. Pitha PM: Introduction: interferon's connection to cancer. Semin Cancer Biol 10: 69-72, 2000.

29. Stojdl DF, Lichty BD, ten Oever BR, Paterson JM, Power AT, Knowles S, Marius R, Reynard J, Poliquin L, Atkins H, Brown EG, Durbin RK, Durbin JE, Hiscott J and Bell JC: VSV strains with defects in their ability to shutdown innate immunity are potent systemic anti-cancer agents. Cancer Cell 4: 263-275, 2003.

30. Sun WH, Pabon C, Alsayed Y, Huang PP, Jandeska S, Uddin S, Platanias LC and Rosen ST: Interferon-alpha resistance in a cutaneous T-cell lymphoma cell line is associated with lack of STAT1 expression. Blood 91: 570-576, 1998.

31. Wagner TC, Velichko S, Chesney SK, Biroc S, Harde D, Vogel D and Croze E: Interferon receptor expression regulates the antiproliferative effects of interferons on cancer cells and solid tumors. Int J Cancer 111: 32-42, 2004

32. Krishnamurthy S, Takimoto T, Scroggs RA and Portner A: Differentially regulated interferon response determines the outcome of Newcastle disease virus infection in normal and tumor cell lines. J Virol 80: 5145-5155, 2006.

33. Fiola C, Peeters B, Fournier P, Arnold A, Bucur M and Schirrmacher V: Tumor selective replication of Newcastle disease virus: association with defects of tumor cells in antiviral defence. Int J Cancer 119: 328-338, 2006.

34. Reichard KW, Lorence RM, Cascino CJ, Peeples ME, Walter RJ, Fernando MB, Reyes HM and Greager JA: Newcastle disease virus selectively kills human tumor cells. J Surg Res 52: 448-453, 1992.

35. Bar-Eli N, Giloh H,Schlesinger M andZakay-Rones Z: Preferential cytotoxic effect of Newcastle disease virus on lymphoma cells. J Cancer Res Clin Oncol 122: 409-415, 1996.

36. Tzadok-David Y, Metzkin-Eizenberg M and Zakay-Rones Z: The effect of a mesogenic and a lentogenic Newcastle disease virus strain on Burkitt lymphoma Daudi cells. J Cancer Res Clin Oncol 121: 169-174, 1995.

37. Chen LL, Yang L and Carmichael GG: Molecular basis for an attenuated cytoplasmic dsRNA response in human embryonic stem cells. Cell Cycle 9: 3552-3564, 2010.

38. Lin R, Yang L, Nakhaei P, Sun Q, Sharif-Askari E, Julkunen I and Hiscott J: Negative regulation of the retinoic acid-inducible gene I-induced antiviral state by the ubiquitin-editing protein A20. J Biol Chem 281: 2095-2103, 2006.

39. Moore CB, Bergstralh DT, Duncan JA, Lei Y, Morrison TE, Zimmermann AG, Accavitti-Loper MA, Madden VJ, Sun L, Ye Z, Lich JD, Heise MT, Chen Z and Ting JP: NLRX1 is a regulator of mitochondrial antiviral immunity. Nature 451: 573-577, 2008

40. Rajput A, Kovalenko A, Bogdanov K, Yang SH, Kang TB, Kim JC, Du J and Wallach D: RIG-I RNA helicase activation of IRF3 transcription factor is negatively regulated by caspase-8-mediated cleavage of the RIP1 protein. Immunity 34: 340-351, 2011.

41. Su ZZ, Sarkar D, Emdad L, Barral PM and Fisher PB: Central role of interferon regulatory factor-1 (IRF-1) in controlling retinoic acid inducible gene-I (RIG-I) expression. J Cell Physiol 213: 502-510, 2007.

42. Romeo G, Fiorucci G, Chiantore MV, Percario ZA, Vannucchi S and Affabris E: IRF-1 as a negative regulator of cell proliferation. J Interferon Cytokine Res 22: 39-47, 2002.

43. Shmulevitz M, Pan LZ, Garant K, Pan D and Lee PW: Oncogenic Ras promotes reovirus spread by suppressing IFN-beta production through negative regulation of RIG-I signaling. Cancer Res 70: 4912-4921, 2010

44. Childs K, Stock N, Ross C, Andrejeva J, Hilton L, Skinner M, Randall R and Goodbourn S: MDA-5, but not RIG-I, is a common target for paramyxovirus V proteins. Virology 359: 190-200, 2007.

45. Picaud S, Bardot B, De Maeyer E and Seif I: Enhanced tumor development in mice lacking a functional type I interferon receptor. J Interferon Cytokine Res 22: 457-462, 2002. 
46. Navarro S, Colamonici OR and Llombart-Bosch A: Immunohistochemical detection of the type I interferon receptor in human fetal, adult, and neoplastic tissues. Mod Pathol 9: 150-156, 1996.

47. Zhou R, Yazdi AS, Menu P and Tschopp J: A role for mitochondria in NLRP3 inflammasome activation. Nature 469: 221-225, 2011

48. Koshiba T, Yasukawa K, Yanagi Y and Kawabata S: Mitochondrial membrane potential is required for MAVS-mediated antiviral signaling. Sci Signal 4: ra7, 2011.

49. Nakahira K, Haspel JA, Rathinam VA, Lee SJ, Dolinay T, Lam HC, Englert JA, Rabinovitch M, Cernadas M, Kim HP, Fitzgerald KA, Ryter SW and Choi AM: Autophagy proteins regulate innate immune responses by inhibiting the release of mitochondrial DNA mediated by the NALP3 inflammasome. Nat Immunol 12: 222-230, 2011.

50. Liu F and $\mathrm{Gu}$ J: Retinoic acid inducible gene-I, more than a virus sensor. Protein Cell 2: 351-357, 2011.

51. Loo YM and Gale M Jr: Immune signaling by RIG-I-like receptors. Immunity 34: 680-692, 2011.

52. Kübler K, Gehrke N, Riemann S, Böhnert V, Zillinger T, Hartmann E, Pölcher M, Rudlowski C, Kuhn W, Hartmann G and Barchet W: Targeted activation of RNA helicase retinoic acid-inducible gene-I induces proimmunogenic apoptosis of human ovarian cancer cells. Cancer Res 70: 5293-5304, 2010.
53. Schlee M, Roth A, Hornung V, Hagmann CA, Wimmenauer V, Barchet W, Coch C, Janke M, Mihailovic A, Wardle G, Juranek S, Kato H, Kawai T, Poeck H, Fitzgerald KA, Takeuchi O, Akira S, Tuschl T, Latz E, Ludwig J and Hartmann G: Recognition of 5 ' triphosphate by RIG-I helicase requires short blunt doublestranded RNA as contained in panhandle of negative-strand virus. Immunity 31: 25-34, 2009.

54. A-Gonzalez N, Bensinger SJ, Hong C, Beceiro S, Bradley MN, Zelcer N, DenizJ, Ramirez C,Díaz M, Gallardo G,De Galarreta CR, Salazar J, Lopez F, Edwards P, Parks J, Andujar M, Tontonoz P and Castrillo A: Apoptotic cells promote their own clearance and immune tolerance through activation of the nuclear receptor LXR. Immunity 31: 245-258, 2009.

55. Fournier P, Arnold A, Wilden H and Schirrmacher V: Newcastle disease virus induces pro-inflammatory conditions and type I interferon for counter-acting Treg activity. Int J Oncol (In press). 